\title{
Engager le dialogue
}

\section{Simonetta Sommaruga ${ }^{a}$, \\ Jacqueline Bachmann ${ }^{b}$}

a présidente de la Fondation pour la protection des consommateurs FPC

b directrice de la Fondation pour la protection des consommateurs FPC
Correspondance:

Fondation pour la protection des consommateurs FPC Monbijoustrasse 61 Case postale

CH-3000 Berne 23

Tél. 0313702424

Fax 0313720027

admin@konsumentenschutz.ch
A l'occasion du premier symposium éthique américain sur le thème de la vaccination infantile, les autorités sanitaires ont communiqué ce qui suit: aux Etats-Unis, les parents se détournent d'autant plus des vaccinations que l'on en propose davantage (actuellement 37 vaccinations infantiles contre 16 maladies). Le scepticisme des parents bien informés en particulier serait en hausse. Par ailleurs, des familles toujours plus nombreuses n'auraient plus les moyens de s'offrir les vaccinations. Le problème principal résulterait du fait que, pendant longtemps, les interrogations de la population face aux vaccinations n'ont pas été prises au sérieux.

Or c'est précisément le but recherché par le guide FPC depuis sa première parution en 2000. Sur la base d'une réflexion critique nuancée quant aux avantages et aux inconvénients de la vaccination, la brochure relève également les interrogations pour lesquelles il existe au moins certaines indications scientifiques sérieuses. Le passé a prouvé qu'une certaine prudence s'impose. Par exemple, seule la pression mondiale de médecins et d'organisations de consommateurs a permis que l'on dispose actuellement de vaccins ne contenant pas de mercure.

Les critiques à nouveau formulées envers le guide de vaccination de la FPC, ainsi qu'à l'égard de la compétence de ses collaborateurs et de son éditeur, montrent bien la difficulté de lancer une réflexion constructive sur ce sujet. Nous le regrettons, en particulier pour les parents concernés. Il faut clarifier en priorité dans quelle mesure un dialogue constructif est possible en matière de vaccination, nous y sommes tout disposés. Nous considérons que le soin apporté par tous les participants à la réalisation du guide répond parfaitement aux «conditions minimum» de la FMH.

\section{Pas d'objection pendant quatre ans}

Il est étonnant de constater combien de temps il a fallu à la FMH, aux sociétés de discipline médicale et à l'Office fédéral de la santé publique (OFSP) pour intervenir. Le guide sur les vaccinations est paru dans sa première édition en 2000. Ce n'est qu'en août 2003, à la quatrième édition (parue en novembre 2002) que l'OFSP s'est exprimé de manière critique à ce sujet pour la première fois. Par la suite, une rencontre a eu lieu le 13 janvier 2004 entre l'OFSP et la FPC. Le compte-rendu de celle-ci, rédigé par l'OFSP, précise: «Il est normal que des recommandations fassent l'objet d'une évaluation critique et l'OFSP approuve cette démarche. Toutefois, l'OFSP critique les informations inexactes.» (Fin de la citation). Aucune de ces informations n'est toutefois mentionnée dans le compte-rendu. La FPC ne comprend pas pourquoi les médecins, la Commission fédérale pour les vaccinations et l'OFSP n'ont jamais réagi à notre guide avant la publication de sa quatrième édition.

Le Bulletin des médecins suisses $\mathrm{n}^{\circ} 35 \mathrm{du}$ 30 août 2006 a publié une critique de la FMH et des sociétés de discipline médicale sur le guide FPC. Pour la première fois, six ans après la parution du guide, l'OFSP a réagi avec une prise de position publique [2].

\section{Exigence de monovaccins}

Enfin, d'autres questions importantes qui nécessitent une clarification sont posées: en dépit d'une liberté de choix de principe en matière de vaccination, les parents considèrent toujours plus qu'ils subissent une obligation dans la mesure où l'on ne trouve plus de monovaccins. Cela peut inciter certains parents à y renoncer totalement.

Depuis des années, la FPC s'engage en conséquence pour que les monovaccins soient à nouveau plus disponibles afin de permettre une véritable liberté de choix en matière de vaccination.

Ces dernières années, on a retiré progressivement les monovaccins du marché au bénéfice de vaccins à plusieurs composants. Le motif invoqué par l'OFSP est la recherche d'une couverture vaccinale la plus complète possible de la population contre un maximum de maladies. Actuellement, la renonciation aux monovaccins génère toutefois des problèmes graves.

La renonciation aux monovaccins implique une restriction de la liberté de choix ressentie comme essentielle par une partie de la population. 
Par exemple pour la rubéole

De nombreux parents souhaiteraient une vaccination ciblée non seulement pour la rubéole, mais également pour la rougeole et les oreillons. Si par exemple leur fille a déjà eu la rougeole, ils souhaiteraient la faire vacciner uniquement contre la rubéole à la fin de sa scolarité. En effet, elle dispose déjà d'une protection naturelle contre la rougeole et les oreillons ne sont pas une maladie dangereuse pour les filles. Tant que l'on trouvera uniquement des vaccins combinés, ils renonceront alors souvent à la vaccination.

\section{Par exemple pour le tétanos}

La vaccination contre le tétanos est efficace et protège d'une maladie grave. Elle bénéfice d'une excellente acceptation au sein de la population. Toutefois, actuellement en Suisse, 10\% des enfants en fin de scolarité ne sont pas suffisamment vaccinés contre le tétanos. Dans certains cantons, la couverture vaccinale est encore nettement plus faible [3]. Cette situation résulte notamment du manque de disponibilité des vaccins infantiles uniquement contre le tétanos (Te) ou le tétanos et la diphtérie (DI-Te) sans le composant de la coqueluche (Pertussis, Per). Lorsque les parents ne veulent pas faire vacciner leurs enfants contre la coqueluche, ils renoncent parfois également à les faire vacciner contre le tétanos puisqu'il n'existe pas de monovaccin.

La FPC est toute disposée à aborder et à débattre ces thèmes avec la FMH.

\section{Références}

1 SSP, SSI, GIPS. Stellungnahme zur Broschüre «Impfen - Grundlagen für einen persönlichen Impfentscheid» des schweizerischen Konsumentenschutzes (6. Auflage). Schweiz Ärztezeitung. 2006;87(35):1485-90.

2 Office fédéral de la sante publique, Division Maladies transmissibles. Prise de position de l'Office fédéral de la santé publique (OFSP). Bull Méd Suisses. 2006;87(37):1596.

3 Office fédéral de la santé publique. Couverture vaccinale en Suisse de 1999-2003. Bulletin OFSP 2006;19:366-71.

\section{Réponse des sociétés de discipline médicale et de la FMH à la réplique de la Fondation suisse pour la protection des consommateurs (FPC)}

\section{Réponse des sociétés de discipline médicale}

Nous sommes heureux que Mme Sommaruga et la FPC soient prêtes à engager le dialogue et nous nous réjouissons d'une collaboration constructive.

Prof. David Nadal, président de la Société suisse d'infectiologie (SSI),

Zurich

Dr Christoph Berger, p.-d., président du Groupe d'infectiologie pédiatrique Suisse (PIGS), Zurich

Dr Pierre Klauser, past président SSP/SGP, Genève

\begin{abstract}
Réponse de la FMH
Madame la Présidente,

Madame la Secrétaire générale,

C'est avec un réel intérêt que la FMH prend acte de votre réponse aux prises de position des sociétés de discipline qui ont examiné votre brochure.

Vous avez très justement relevé que les objections des sociétés de spécialistes consultées visent à soutenir le caractère scientifique et impartial d'une information publique sur la vaccination et pas à s'opposer au principe du droit à une information libre et éclairée des consommateurs - information que le corps médical soutient ardemment.
\end{abstract}


Dans le champ de la prévention et de la promotion de la santé, il est incontestable que le domaine de la vaccination est un lieu sur lequel convergent des contraintes politiques de santé publique, des éléments de la réalité scientifique, des pressions économiques et les intérêts de la liberté individuelle. Cela conduit inévitablement à une tension structurelle qui ne sera probablement jamais entièrement réduite en raison entre autres de ses composantes idéologiques et émotionnelles. Ces derniers paramètres ont d'ailleurs certainement joué un rôle dans le temps de latence que vous pointez dans votre réaction.

Cela dit, cette tension ne doit pas empêcher une réflexion approfondie et conjointe sur ce qui est fondé et sur ce qui ne l'est pas dans le domaine de la vaccination. Vous proposez d'ailleurs déjà une piste. La FMH, appuyée par la position des sociétés de discipline qu'elle a consultées, accueille ainsi très favorablement la proposition de dialogue que vous formulez. Ce dialogue devrait nous conduire à l'élaboration d'une future brochure, dont le contenu visera précisément une information neutre et éclairée des consommateurs sur les questions liées à la vaccination. Le résultat permettra, nous l'espérons, de répondre à la demande d'une information permettant l'exercice du libre choix des consommateurs sur le terrain de la prévention des maladies infectieuses.

La FMH soutient donc la rencontre de la Fondation pour la protection des consommateurs et des sociétés de spécialistes concernées. Elle fera le nécessaire afin que ces discussions puissent débuter prochainement et qu'elles répondent à un objectif commun.

Dans l'attente de vous rencontrer pour une première entrevue, nous vous prions de recevoir, Madame la Présidente, Madame la Secrétaire générale, l'expression de nos sentiments les meilleurs.

Dr Jacques de Haller, président de la FMH

Dr R. Raggenbass, membre du Comité central, responsable de la division santé prévention

En complément à la réponse de Mme Simonetta Sommaruga et de Mme Jacqueline Bachmann, les Docteurs Martin Hirte et Hansueli Albonico ont également rédigé une réponse que nous ne manquerons pas de publier prochainement dans ce Bulletin. 